\title{
Intelligent expression the transceiver system design ideas based on NFC technology
}

\author{
Wei Wei ${ }^{1, a}$ \\ ${ }^{1}$ Wuhan City Vocational College, Wuhan, Hubei Province, China \\ a13317355@qq.com
}

Keywords: NFC technology, Intelligent, Express delivery, Sending and Receiving system

\begin{abstract}
The widely application of the intelligent logistics is based on Internet of things, based on the use of advanced information collection, information processing, information flow and information management technology, complete including transportation, warehousing, distribution, packaging, loading and unloading and so on many basic activities of the whole process of moving goods from suppliers to demanders, provide the supplier to maximize profits, to provide the best service to the buyer, the least consumption of natural resources and social resources at the same time, the maximum to protect the ecological environment of the whole intelligent social logistics management system. This paper will be collected in order to enhance express convenient degree, help property at the front desk and buyers to provide convenient, for the purpose of designing a intelligent since the transceiver system based on NFC, in order to improve the efficiency of the Courier and promote the development of express industry.
\end{abstract}

\section{Introduction}

With the rapid development of social economy and the overall progress of science and technology, the rapid development of computer business, on the basis of computer and communication technology of information system is in the booming period. Along with the economic and cultural levels increased significantly, the requirements of the quality of life and working environment more and more is also high. In the commodity economy society, in order to manage a large number of goods, logistics management problem is increasing [1]. With the large increase in the variety of goods, cumbersome manual operation, the management difficulty is also more and more big, how to optimize the day-to-day management of logistics has become a popular topic. In today's rapid development of computer, the computer is a tool used in the daily management of the logistics information processing is inevitable trend, and it will also bring unprecedented change for logistics management, it can bring unexpected benefits, while also offers unlimited potential for the rapid development of enterprises [1]. By using the computer management information system has become a scientific logistics management and an important symbol of modernization, it is to the enterprise management to the obvious economic benefits and social benefits.

\section{Modern logistics are faced with the problem}

Though the modern logistics industry has been preliminary realized electronic, but also there are insurmountable difficulties. Especially in the recent 20 years, the new logistics services, such as the third party logistics, lean logistics mode appear constantly, such as is necessary for the existing information technology can provide a more intelligent, more interconnected, more precise support and services. And existing electronic logistics platform, whether it has universal bar code technology, the EDl technology still has a lot of, can't meet this need.

Connectivity is not sufficient. The connectivity of logistics system is multifaceted. First of all, the lacks of necessary standards support e-logistics system [2]. The benefits of using ED1 are obvious, but the subsequent standards war has never ceased. Such as the standardization of bar code is one of the biggest difficulty in logistics distribution. Barcode standardization problem is a standard, it is difficult to compatible with each other, should have a unified standard is not unified; The second is the development of standards seriously lags behind the economic development speed, there are a lot 
of coding standard classification is not clear, not only can't play the role of improving efficiency, instead affects the velocity of the item, increase the processing burden of the system; Three specification standard is not popular, a lot of applications for the use of bar code is coming into its own, the man-made barriers to "standard" [2]. Do not have a unified standard of the consequences can be rather serious, to let the goodness of EDl originally envisaged is difficult to quickly implement.

In addition, heterogeneity is also difficult to improve efficiency of e-logistics one main reason, including heterogeneous and heterogeneous network equipment [2]. Logistics system in a variety of equipment, confused or electronically information degree is differ, the existing computer system cannot integrate these devices in an efficient logistics system, processing bottleneck caused by much, a lot of processing link constraint each other, unable to exert the maximum efficiency of system. Although at the same time, a lot of information collected in computer system, but has not been fully utilized, the reason is that the information in the electronic logistics system is not fully communication and sharing, caused great waste of resources.

Perception is not timely, incomplete. Modern logistics industry requirements to don't ask, don't break every link and process monitoring and management of logistics, real time control of the state and the information of the item can at the same time, and to make timely and accurately reflect the various events. And e-logistics technology cannot meet this need [1]. Using barcode only guarantee, for example, in certain aspects such as population and to identify the location, and once you object to leave the link, such as warehousing, cannot automatically positioning and tracking of objects [3]. When needed from the warehousing of parcel or pick out a particular item in the parcel, must be artificial all barcode scanning nearby, is both time-consuming and laborious also can't guarantee success.

In addition, the current single electronic logistics information collection means. Bar code can only reflect the items in the system default corresponding information, cannot report real-time status of the item. Item damage happens, if the barcode is not damaged, probably until (till) the final customers can be found there. In addition, as the logistics process in the food industry need to temperature, humidity, pressure, illumination, etc [3]. The diversity of information collection, and can make corresponding control in real time. These requirements are electronic logistics existing means and technology cannot achieve.

The lack of an intelligent computing support and services. E-logistics although adopted many large commercial software, used to improve the level of logistics system, the intelligent processing, but the application of intelligent degree is low, most intelligent function of logistics software limitations in cost control, resource allocation and decision support, is not the comprehensive utilization of intelligent equipment to meet the user a variety of customized, personalized needs [3]. At the same time, the synergistic effect of e-logistics, most enterprise system individually, can't play to the advantages of cooperation. People need more wisdom; to integrate resources of the whole logistics industry, more delicate and dynamic management of logistics activities, raise the level of resource utilization and productivity.

\section{NFC transceiver system design}

Intelligent logistics. With the emergence of the Internet of things, the logistics industry has ushered in a new development opportunity. The concept of Internet of things, the first is to ring in the logistics industry [4]. Modern logistics system in order to overcome the shortcoming of e-logistics, hope that the use of information generating equipment, such as radio frequency identification devices, sensors and global positioning system (GPS) device such as a variety of combined with Internet and form a huge network, and can in the intelligent instrumentation of the logistics network of logistics management.

The characteristics of the intelligent logistics. The developments of intelligent logistics are present accurate, intelligent, collaborative features. Precision comes from lean construction of the construction industry. Is the purpose of lean construction by precise planning, design and supply, using the whole process of product control and information feedback, to minimize the waste of resources, to maximize profit value of construction management approach. Precision of logistics 
demand and zero waste is minimizing the total cost [4]. Specifically, intelligent logistics system in the future, because of the instrumentation intelligent information processing system and the general use of intelligent equipment, logistics enterprise managers want to achieve procurement, inbound, outbound, transfers, assembling, transportation link the precise management, such as inventory, transportation and manufacturing costs to a minimum, at the same time each link may produce waste reduced to zero.

In addition to reduce costs and reduce the waste of the basic goals, the future need to collect real-time information intelligent logistics system, by using the Internet of things system processing, and consulting services for end users to provide quality information, provide the best strategy, support for the logistics enterprises. To be sure, the Internet provides the intelligent processing of intelligent logistics multifaceted support, in addition to the use of the existing ERP and other commercial software for integrated planning, management and decision support, the future of smart logistics more should pay attention to use equipment and network itself more intelligent service [5]. It can be imagined, the future of equipment should not only provide marking and the function of information collection, at the same time can also undertake a broader processing functions. With smart logistics, the logistics enterprises can optimize the allocation of resources, business processes, and to provide value-added logistics services to end users, broaden the scope of business, finally realize the profit maximization.

NFC system. In the intelligent logistics, objects or user identity is the most important information. Internet scholars also had this kind of consciousness, such as the new IPv6 theory can give every star dust on earth an IP address [5]. Since the next generation of Internet of the information platform of the core network is the Internet, so, every object in the Internet of things, the identity of the members, whether can also like IP address, we will establish a unified data format, complete analytical framework and comprehensive geographical coverage.

NFC transceiver system. From the middle of the 20th century electronic technology, people have been pursuing the use of this technology to achieve faster, more convenient communication. Telegraph, telephone, fax, have been widely applied to the business, they initially in order to assist paper mainly trade measures; a large number of commercial activities need to pass the paper document to complete. The emergence of computer and network, real business into the digital era, it is shown in Fig. 1.

Intelligent express business is developing rapidly, city deployment and operations management will be more and more, hope to these intelligent express mail box operating unit for unified management in the form of wireless remote terminal node, self-help express ark wireless networking and remote operations management demand arises at the historic moment [6]. Based on $3 \mathrm{~g} / 4 \mathrm{~g}$ industrial-grade routers and M2M cloud management platform provide self-service delivery cabinet wireless networking management for industry customers one-stop solution, completely solve the "last kilometer" at the end of the logistics wireless networking and remote terminal management problems.

Intelligent express is a based on Internet of things, able to goods (express), which can identify the staging, monitoring and management of equipment. And express tank monitoring platform system intelligent express delivery box [2]. Express tank monitoring platform to this system, each express delivery box for unification management (such as express delivery of information, express information, user information, etc. and to handle all kinds of information integration analysis. Will express Courier service after the designated place, need to be deposited in the express delivery box, the system will automatically send a text message for the user, including the pick-up address and verification code, the user at a convenient time to reach the terminal input validation code can express, it is shown in Fig. 2. . 


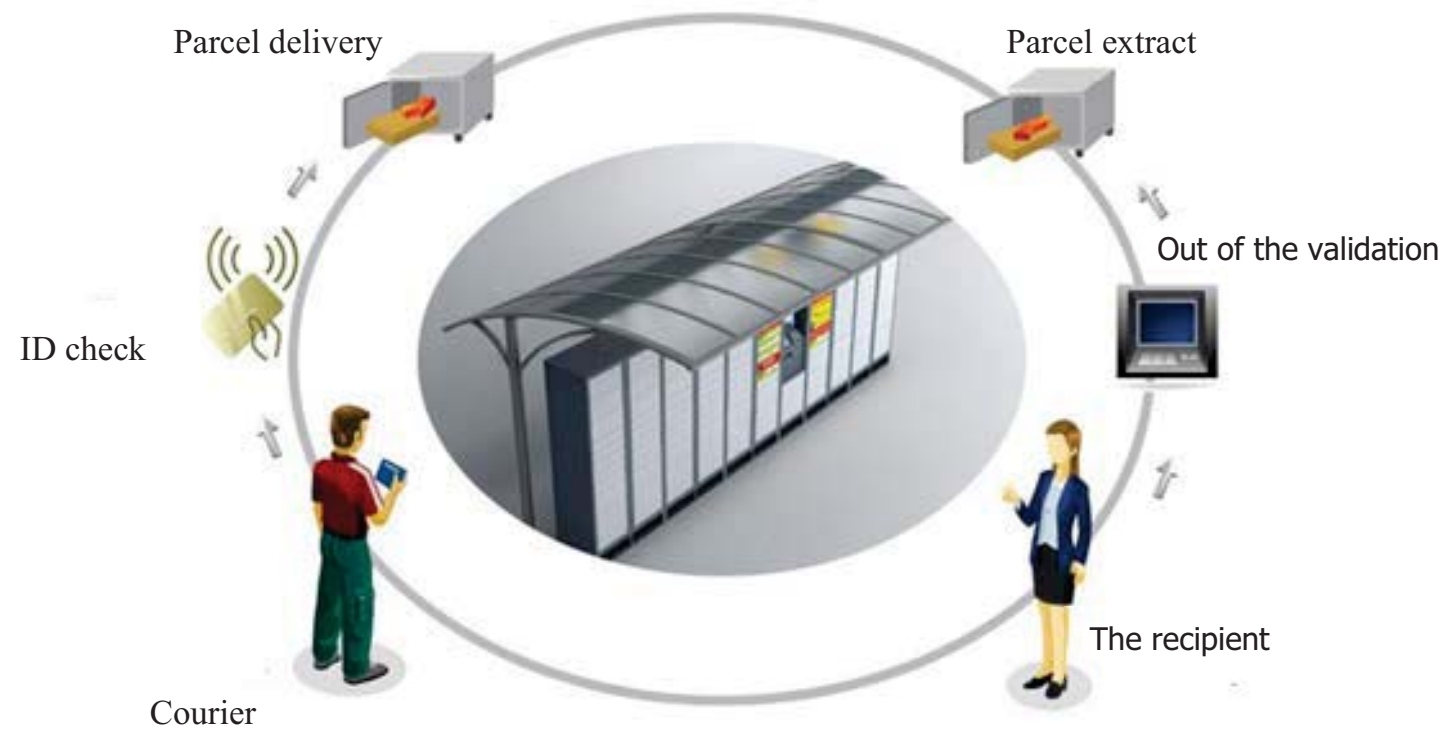

Fig. 1 NFC transceiver system

NFC intelligent delivery system by the NFC tags, data acquisition subsystem and the center of the server subsystem network topology are [6]:

NFC tags: different equipment and place USES different kinds of labels, to ensure the reliable use of the label.

The data acquisition subsystem, data acquisition subsystem by Smartphone with (NFC) and NFC express of the client. System personnel mobile phone number for the account, login system, can read NFC tags. When after delivery person records the express content click "submit" button (if it is a security guard patrolling is not required) to the delivery point express the content of real-time database server via $3 \mathrm{G} / 4 \mathrm{G}$ networks. A Courier delivery point is accomplished; express the client will be prompted to enter the next express delivery point, interface as shown in the figure below.

The center server subsystem consists of communication server, database server and a WEB server, realize the data receiving, storage, analysis, and user management, statement analysis, and other functions. Central server subsystem
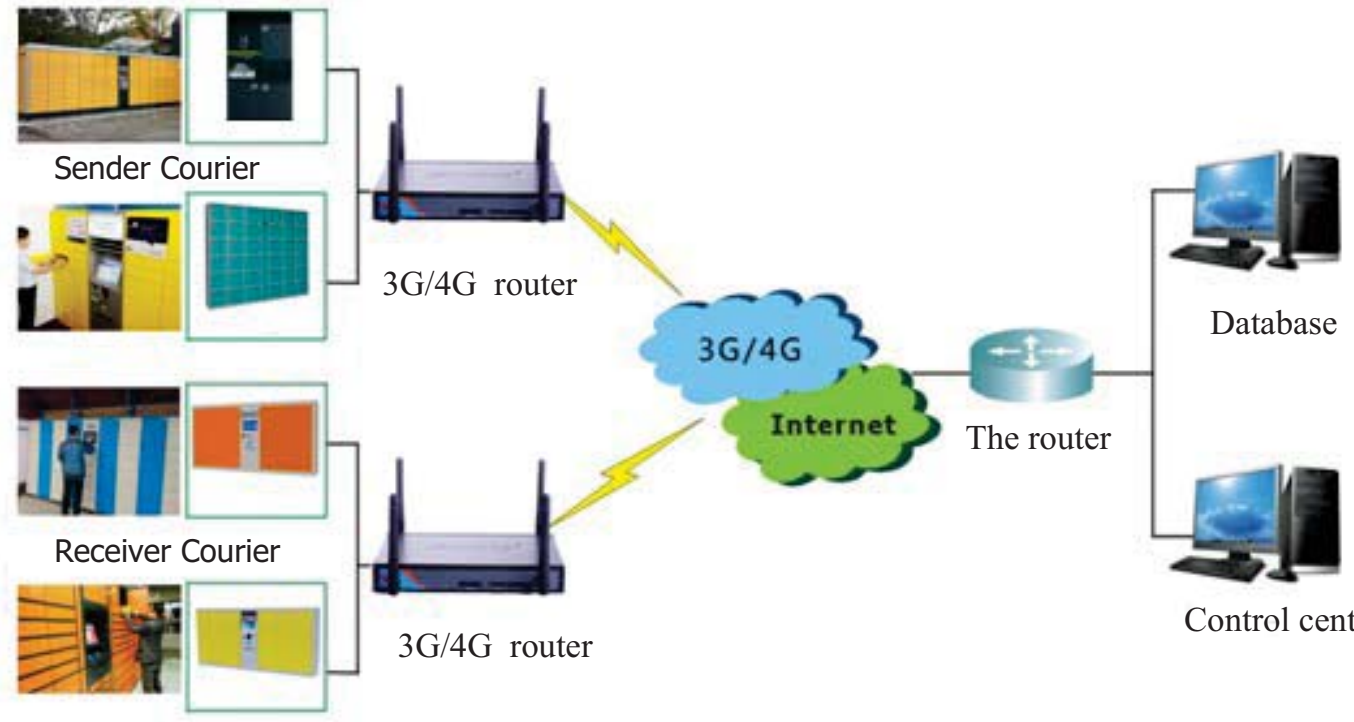

Fig. 2 Network topology of NFC expresses system

Product characteristic features. The core equipment of this system is $3 \mathrm{G} / 4 \mathrm{G}$ for self-help express ark and enterprise business platform to establish link transport channel, the product is a feature of industrial products, wireless network communication using 64 - bit high-performance 
industrial-grade communications MIPS processor, based on RTOS of embedded real-time operating system software support platform, the system integrates the whole series from logical link layer to application layer communication protocol, support static and dynamic routing, can provide users with security, high-speed, stable and reliable routing forwarding wireless router network, various protocols help self-service delivery tank terminal to realize the $3 \mathrm{G} / 4 \mathrm{G}$ wireless networking and remote network access management services [7].

Using hardware and software watchdog and multistage link detection mechanism, fault automatic detection, automatic recovery ability, ensure stable and reliable equipment operation, help logistics enterprise to realize "24 hours" delivery service, take, hair pieces of information such as real-time uploaded to the enterprise background.

A strong remote $\mathrm{M} 2 \mathrm{M}$ management platform, real-time management, hierarchical management, traffic control, access control, statistical analysis, batch export, authority certification, alarm functions, communications security, large quantities of management convenient and efficient, reduce operating costs.

Optional GPS/Beidou positioning, supporting the precise location query function, save maintenance cost. 4, the product can meet the demands of industrial design, and to high/low temperature, high humidity, interference in the outdoor environment and stable operation.

\section{Summary}

Intelligent logistics have mapped the beautiful blueprint for the logistics enterprise. Between dream and reality, however, there is a short journey, about the future of the development of the logistics instrumentation envisaged a lot, but many difficult to achieve, too. The technology of intelligent degree is not high, content also needs to improve equipment utilization, and network construction is the basis of Internet of things not to cover the each link of logistics. This is the challenge of intelligent logistics development and opportunity. Borrow the NFC technology will be an effective way to solve the bottleneck of e-commerce development, become the main driver of the development of logistics industry the next revolutionary.

\section{Acknowledgement}

Fund project: Wuhan city Colleges and universities production research project of 2016 Wuhan Education Bureau. (Project ID: CXY201638).

\section{References}

[1] K. Ch. Ke, The current situation of the modern logistics industry development in China, The countermeasures of modern economy, 2008, vol. 9, pp. 80-82.

[2] X. J. Zhou, Express the development and research of engineering college in Shenyang, Shenyang institute of engineering, 2013, vol. 3, pp. 32-36.

[3] J. T. Liu, The campus express new pattern research, Wuhan commercial service college journal, 2013, vol. 1, pp. 53-57.

[4] N. X. Wang and Sh. Ch. Lin, "90 s" College students and intelligent express terminal, Xinhua net frequency of Fujian, 2014, vol. 10, pp. 60-64.

[5] M. T. Zhou, Development status and trend of intelligent logistics system at home and abroad, The railway procurement and logistics, 2007, vol. 2, pp. 23-27.

[6] Y.M. Mao, The development trend of intelligent logistics system, Logistics and purchasing in China, 2014, vol.6, pp.12-16.

[7] Y. M. Mao, The development trend of intelligent logistics system, Logistics and purchasing in China, 2014, vol. 6, pp. 12-16. 